\title{
Dipole Polarizability of a Trapped Superfluid Fermi Gas
}

\author{
A. Recati, I. Carusotto, C. Lobo, and S. Stringari* \\ Dipartimento di Fisica, Università di Trento and CNR-INFM BEC Center, I-38050 Povo, Trento, Italy
}

(Received 31 May 2006; published 9 November 2006)

\begin{abstract}
The polarization produced by the relative displacement of the potentials trapping two spin species of a dilute Fermi gas with $N_{\uparrow}=N_{\downarrow}$ is calculated at unitarity by assuming phase separation between the superfluid and a polarized phase at zero temperature. Because of the energy cost associated with pair breaking, the dipole polarizability is strongly quenched and exhibits important deviations from the ideal gas behavior even for nonlinear displacements of the order of the size of the atomic cloud. The behavior in the presence of different trapping frequencies (monopole polarization) for the two spin species is also discussed. Our results suggest new experimental perspectives to explore the quantum phases of interacting Fermi gases.
\end{abstract}

It is well known that Fermi superfluids cannot be polarized by an external magnetic field unless the field exceeds a critical value. This effect is directly associated with the occurrence of a gap in the spin excitation spectrum and is the consequence of the existence of pairs. In a dilute gas the nature of these pairs depends crucially on the value and the sign of the $s$-wave scattering length. For negative and small values of the scattering length the pairs coincide with the Cooper pairs of BCS superconductivity. If the scattering length is instead positive and small, they can be identified with real molecules which, due to their bosonic nature, undergo Bose-Einstein condensation (BEC). With the aid of Feshbach resonances it is now possible to experimentally control the transition between the BCS and BEC regimes and to investigate the challenging unitary regime where the scattering length is much larger than the interparticle distance. The effect of polarization on these novel quantum phases has already been the object of experimental [1-3] and theoretical [4-6] investigations. In particular, the polarization has been shown to give rise to a phase separation between superfluid and nonsuperfluid components, although the detailed structure of the phase separation is still far from being completely understood.

The purpose of this Letter is to investigate directly the nonlinear static response of these systems to a spindependent external field, taking advantage of the fact that the trapping geometry suggests a natural way to generate an effective, position dependent, magnetic field. In other words, we propose to study the system from a new viewpoint, instead of investigating the quantum phases as a function of the total population imbalance, we study them as a function of the conjugate variable, the difference in the chemical potential of the two species (see below). This is achieved, for example, by an adiabatic separation of the external potentials confining the two spin species. If the gas is noninteracting, the two spin clouds will move rigidly in opposite directions giving rise to a dipole polarization per particle,

$$
D(d)=\frac{1}{N} \int x\left(n_{\uparrow}-n_{\downarrow}\right) d \mathbf{r},
$$

equal to one-half the distance separating the two wells. In Eq. (1) $N$ is the total particle number and $2 d$ the relative displacement of the two trapping potentials. If instead the system is interacting and superfluid, it will exhibit a strong resistance to dipole polarization. At zero temperature a crucial consequence is that the system cannot have out of phase collective oscillations and can be polarized only via nonlinear effects [7].

In order to calculate the effects of the local polarization induced by the displacement of the confining potentials we will make use of the phase diagram of uniform matter and the corresponding equations of state which are available in some relevant regimes, the equilibrium between the different phases being obtained by imposing that the corresponding pressures be equal. In the following we will mainly focus on the unitary regime where the equation of state takes a universal form. In the unpolarized phase the system is superfluid at zero temperature and its equation of state is

$$
\mu_{s}=\frac{\hbar^{2}}{2 m} \xi\left(6 \pi^{2} n\right)^{2 / 3},
$$

where $n=n_{\uparrow}=n_{\downarrow}$, exhibiting the same power law density dependence as in the noninteracting gas. The dimensionless parameter $\xi$ accounts for the role of interactions. Its value, evaluated with $a b$ initio Monte Carlo simulations, is $\xi \simeq 0.44$ (see, e.g., [8]). In the presence of polarization we will assume that the system exhibits phase separation between an unpolarized superfluid phase $(s)$ governed by the equation of state (2) and a fully polarized phase $(p)$ where interactions can be ignored and the equation of state is given by the noninteracting expression

$$
\mu_{p}=\frac{\hbar^{2}}{2 m}\left(6 \pi^{2} n\right)^{2 / 3},
$$

with $n$ the density of the only species present in such a 
phase. This is the simplest assumption on resonance. The more complicated possibility of an intermediate nonsuperfluid phase where the two components coexist with different densities has been recently explored theoretically $[4,6]$ (see also [9]) and experimentally [3].

In terms of the chemical potentials $\mu_{s}$ and $\mu_{p}$ the equilibrium between the pressures of the two phases can be written in the form

$$
2 \mu_{s}=(2 \xi)^{3 / 5} \mu_{p} .
$$

It is useful to represent the equilibrium condition (4) in the $\mu$ - $h$ phase diagram, where $\mu=\left(\mu_{\uparrow}+\mu_{\downarrow}\right) / 2$ is the average value of the two chemical potentials while $h=\left(\mu_{\uparrow}-\right.$ $\left.\mu_{\downarrow}\right) / 2$ is an effective magnetic field fixed by the difference between the two chemical potentials. From general thermodynamic considerations [5] we find

$$
\mu_{s}=\frac{1}{2}\left(\mu_{\uparrow}+\mu_{\downarrow}\right),
$$

so that for $h>0$, where $\mu_{p}=\mu_{\uparrow}$, the phase separation between the superfluid and the polarized phase in the $\mu-h$ plane is described by the straight line

$$
\mu=\frac{(2 \xi)^{3 / 5}}{2-(2 \xi)^{3 / 5}} h
$$

The trapping introduces a position dependence in the chemical potentials of the two atomic species that can be evaluated according to the local density expressions

$$
\mu_{\uparrow \downarrow}(\mathbf{r})=\mu_{\uparrow \downarrow}^{0}-V_{\uparrow \downarrow}(\mathbf{r}) .
$$

Equation (7) allows us to calculate the $\mathbf{r}$ dependence of the chemical potential of both the superfluid and polarized phases. The equilibrium condition (4) then becomes an equation characterizing the $\mathbf{r}$ dependence of the surface separating the two phases. The constant values $\mu_{\uparrow \downarrow}^{0}$ are determined by imposing the proper normalization on the spin-up and spin-down densities.

If the trapping potential is the same for the two species $\left(V_{\uparrow}=V_{\downarrow}\right)$, then the effective magnetic field $h$, which differs from zero if $N_{\uparrow} \neq N_{\downarrow}$, is independent of position, and by varying $\mathbf{r}$, we are consequently exploring the $\mu$ - $h$ phase space at constant $h$. This is the case of all the configurations so far investigated in the literature. If instead the two external potentials are different, the magnetic field $h$ will be position dependent. We will discuss, in particular, the case of a dipole displacement of the harmonic traps

$$
V_{\uparrow \downarrow}(\mathbf{r})=\frac{1}{2} m\left[\omega_{\perp}^{2} r_{\perp}^{2}+\omega_{x}^{2}(x \mp d)^{2}\right],
$$

with $r_{\perp}=\sqrt{y^{2}+z^{2}}$. Furthermore, we will assume the system to be globally unpolarized $\left(N_{\uparrow}=N_{\downarrow} \equiv N / 2\right)$ so that, for symmetry reasons, $\mu_{\uparrow}^{0}=\mu_{\downarrow}^{0} \equiv \mu_{0}$. Thus $h$ is $x$ dependent, and in the $\mu-h$ plane, we have

$$
\begin{aligned}
\mu(\mathbf{r}) & =\mu_{0}-\frac{1}{2} m \omega_{x}^{2} d^{2}-\frac{1}{2} m\left(\omega_{x}^{2} x^{2}+\omega_{\perp}^{2} r_{\perp}^{2}\right), \\
h(\mathbf{r}) & =m \omega_{x}^{2} x d .
\end{aligned}
$$

By varying $x$, we then explore the phase diagram along a parabolic trajectory.

It is worth noticing that for any value of the displacement $d$ the effective potential $\left[V_{\uparrow}(\mathbf{r})+V_{\downarrow}(\mathbf{r})\right] / 2$ felt by the system in the superfluid phase has a minimum at $x=0$ giving rise to a superfluid density profile symmetric in the $x$ direction:

$$
n_{s}=\frac{1}{6 \pi^{2}}\left(\frac{2 m}{\xi \hbar^{2}}\right)^{3 / 2}\left(\mu_{0}-\frac{1}{2} m \omega_{\perp}^{2} r_{\perp}^{2}+\frac{1}{2} m \omega_{x}^{2}\left(x^{2}+d^{2}\right)\right)^{3 / 2} .
$$

The potentials trapping the spin-up and spin-down components have instead minima at $x= \pm d$, respectively, thereby favoring the formation of a polarized configuration with density

$$
n_{\uparrow \downarrow}^{p}=\frac{1}{6 \pi^{2}}\left(\frac{2 m}{\hbar^{2}}\right)^{3 / 2}\left(\mu_{0}-\frac{1}{2} m \omega_{\perp}^{2} r_{\perp}^{2}-\frac{1}{2} m \omega_{x}^{2}(x \mp d)^{2}\right)^{3 / 2} .
$$

The boundary separating the two density profiles is fixed by the condition (4) of mechanical equilibrium and is characterized by the typical geometry of Fig. 1, where, for the sake of simplicity, we have shown a $2 \mathrm{D}$ cut. For example, for $x>0$ we have $\mu_{p}=\mu_{\uparrow}$ and the boundary between the superfluid and the spin-up normal component is fixed by the condition

$$
(x+\alpha d)^{2}+\lambda r_{\perp}^{2}=R_{x}^{2}+\gamma d^{2},
$$

where we have introduced the radius $R_{x}=2 \mu_{0} / m \omega_{x}^{2}$ of the polarized component, $\lambda=\omega_{\perp} / \omega_{x}, \alpha=(2 \xi)^{3 / 5} /[2-$ $\left.(2 \xi)^{3 / 5}\right]$, and $\gamma=4\left[(2 \xi)^{3 / 5}-1\right] /\left[2-(2 \xi)^{3 / 5}\right]^{2}$. We find that, for any value $d<R_{x}^{0}$, where $R_{x}^{0}=a_{x}^{\text {ho }}(24 N)^{1 / 6}$ is the Thomas-Fermi radius of the noninteracting cloud, with $a_{x}^{\text {ho }}=\sqrt{\hbar / m \omega_{x}}$, there is equilibrium between the super-

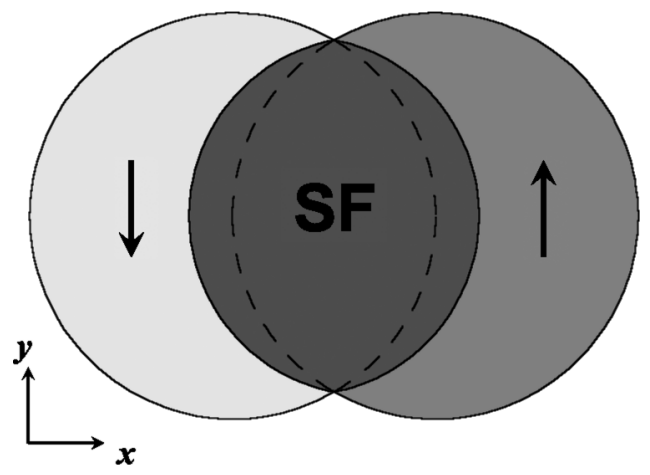

FIG. 1. Sketch of the dipolar configuration in the $x-y$ plane, for $z=0$ (see text). The gray and light gray sectors are the spin-up and spin-down polarized clouds, respectively. The dark gray central part is the superfluid (SF) gas. 
fluid and the polarized phases. Conversely, if $d>R_{x}^{0}$, the superfluid is absent and the two polarized clouds are separated in space.

Starting from results (10) and (11) it is possible to calculate the dipole polarization (1) as a function of the displacement $d$ of the traps. The spin-up (-down) densities entering the integral (1) are given by the sum of the superfluid and polarized components: $n_{\uparrow \downarrow}=n_{s}+n_{\uparrow \downarrow}^{p}$. Since the superfluid density is $x$ symmetric, it does not contribute to the integral and the dipole moment turns out to be reduced with respect to the value $D=d$ predicted in the absence of interactions. In particular, when $d \ll R_{x}^{0}$ the induced dipole polarization takes the simple power law behavior

$$
D(d) \rightarrow \frac{2^{10}}{45 \pi} R_{x}^{0}\left(\frac{d}{R_{x}^{0}}\right)^{5 / 2} \frac{\xi^{9 / 8}}{\left[2-(2 \xi)^{3 / 5}\right]^{5 / 2}},
$$

revealing explicitly that the response $D(d) / d$ vanishes in the linear limit $d \rightarrow 0$. Figure 2 shows that, due to superfluidity, the induced dipole moment deviates significantly from the noninteracting value $D=d$ at distances of the order of the size of the atomic cloud. At the same time, the figure, as well as Eq. (13), shows that the induced dipole is always different from zero even for small displacements, revealing the absence of a true gap. These two features characterize the behavior of the trapped superfluid at unitarity. Actually, near the border of the cloud, the gap, being proportional to the Fermi energy, becomes smaller and smaller and even a tiny magnetic field can induce a finite, although small, polarization.

The polarization of the gas becomes more and more pronounced on the BCS side of the resonance. In fact, in this case the gap decreases exponentially when we reach the border, and as a consequence, we can more easily

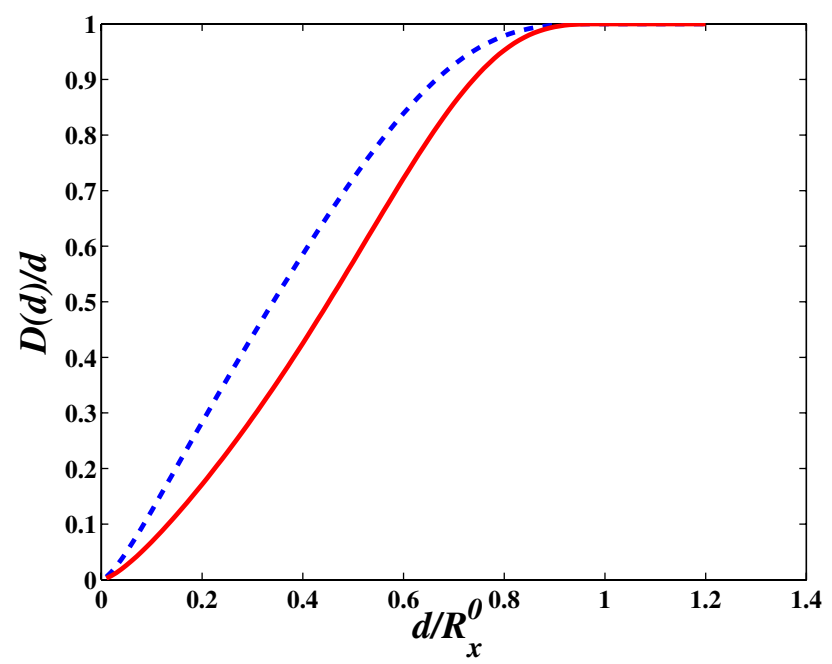

FIG. 2 (color online). Normalized induced dipole polarizability $D(d) / d$ vs displacement $d$ of the trapping potential. The displacement is given in units of the Thomas-Fermi radius $R_{x}^{0}$. Solid line: prediction using the Monte Carlo value, $\xi \simeq 0.44$. Dashed line: prediction within BCS approach, $\xi=0.58$. polarize the medium by breaking Cooper pairs. For a trapped gas in the deep BCS regime we expect that the induced dipole moment approaches the value $D=d$ at small displacement distances, which can be estimated using energy arguments as $d \sim R_{x}^{0} \sqrt{\Delta / \epsilon_{F}}$, where $\Delta$ is an average value of the superfluid gap.

On the BEC side of the resonance, where molecules are formed at small density, we instead expect a very different behavior, which can be described using 2-body physics. In fact, in this case the gap remains finite even near the surface where it is fixed by the binding energy $\hbar^{2} / m a^{2}$ of the free molecules. The polarization will be zero until the value of the displacement is such that the molecular potential energy increase $\sim m \omega_{x}^{2} d^{2}$ is of the order of the molecular binding energy. This corresponds to separation distances of the order of $d / R_{x}^{0} \simeq 1 / k_{F} a$. In the deep BEC regime, where $k_{F} a \ll 1$, we therefore expect that the system will never be polarized unless the displacement is much larger than the size of the cloud [10].

In conclusion, the transition from the BCS to the BEC regime is predicted to reveal a dramatic behavior of the induced dipole moment, which exhibits a transition from a quasi-ideal regime where the gas is easily polarized and is affected only by superfluidity at small displacements (deep $\mathrm{BCS}$ ), to a regime where the rigidity of molecules is strong and the system is not easily polarized by the separation of the trapping potentials (deep BEC).

In Fig. 2 we have also shown the prediction (dashed line) for the induced dipole polarization calculated using the phase diagram of the BCS approach, which is known to provide a semiquantitative description of the equation of state at unitarity. In our approach the differences are due to the different value for the interaction parameter $\xi$ whose value is 0.58 instead of the correct value 0.44 . The different curves reveal the sensitivity of the dipole polarization curve to the proper description of the equation of state. They are easily interpreted since the smaller the value of $\xi$, the more favorable the superfluid phase and, consequently, the smaller the polarizability. In the BCS approach, in Fig. 1 the superfluid part would appear inside the dashed contour. The BCS approach predicts also that the phase diagram includes a further, normal-mixed phase [6] which is simply a noninteracting gas of spin-up and spin-down components. At unitarity we then predict a transition from a superfluid to a mixed-normal phase and from a mixednormal to a polarized phase as we move from the center to the border of the cloud. The inclusion of this additional phase, within the BCS model, does not result in any visible effect in the physical quantity $D(d)$ [9].

Separating the two trapping potentials and inducing a dipole moment is not the only way to polarize a $N_{\uparrow}=N_{\downarrow} \equiv$ $N / 2$ system. Another procedure consists in modifying the trapping frequencies of the two spin species, i.e., by choosing trapping potentials of the form

$$
V_{\sigma}(\mathbf{r})=\frac{1}{2} m\left(\omega_{\sigma, \perp}^{2} r_{\perp}^{2}+\omega_{\sigma, x}^{2} x^{2}\right), \sigma=\uparrow, \downarrow
$$


with, in general, $\omega_{\uparrow, i} \neq \omega_{\downarrow, i}$ for $i=x, \perp$. This gives rise to a relative compression of the two spin clouds. In the following we consider the simplest case of isotropic trapping, for which the above procedure corresponds to inducing a monopole polarization where the radii of the two species take a different value. For a noninteracting gas we find $R_{\uparrow}^{0}-R_{\downarrow}^{0}=\left(a_{\uparrow}^{\text {ho }}-a_{\downarrow}^{\text {ho }}\right)(24 N)^{1 / 6}$. In the presence of interactions the behavior is quite different. In particular, even for large differences between the trapping frequencies the system remains fully superfluid and does not exhibit monopole polarization unless the value of the interaction parameter $\xi$ is larger than 0.5 . This behavior is well understood by a simple energetic argument. If the trapping frequency of one of the two spin species, say, the spin-up component, is much smaller than the one of the other component, then the configuration with polarization would consist of two noninteracting, practically nonoverlapping clouds with very different radii. The energy of this configuration would be $E^{p} / N=(3 / 4) \hbar \omega_{\downarrow}(3 N)^{1 / 3}$, the contribution of the spin-up component being negligible. Conversely, the superfluid, feeling a confining potential with $\omega_{s}^{2}=\left(\omega_{\uparrow}^{2}+\omega_{\downarrow}^{2}\right) / 2 \simeq \omega_{\downarrow}^{2} / 2$, has an energy equal to $E^{s}=E^{p} \sqrt{2 \xi}$. This is smaller than $E^{p}$ since $\xi=0.44$. In other words, at least within the "two phase description," the system always prefers to form pairs and remain superfluid rather than giving rise to a phase separation as happens in the dipole case. The effect of the asymmetric trapping is a change in the radius of the atomic cloud and in the collective oscillations as now the superfluid feels a different effective trapping frequency. Notice that this peculiar behavior is typical of the unitary regime and of the BEC side of the resonance. On the BCS side, one would expect a partial polarization of the system, since the energetic gain associated with the superfluid phase is smaller than at unitarity.

The analysis of the dipole (and monopole) configuration has been here carried out at unitarity within the easiest assumption of phase separation between a totally polarized normal Fermi gas and a fermionic superfluid and with $N_{\uparrow}=N_{\downarrow}$. The generalization of our approach to $N_{\uparrow} \neq N_{\downarrow}$ configurations is straightforward. Furthermore, the inclusion of additional phases, resulting from the availability of more sophisticated microscopic theories [9], as well as of surface tension effects could be naturally accounted for in the calculation of the dipole polarization.

Concerning the experimental feasibility of the measurement of the polarizability a conditio sine qua non is the possibility of producing spin-dependent trapping potentials. This in principle is feasible by profiting of the different polarization of the electronic spin in the two hyperfine states of the Fermi gas and working with magnetic gradients and/or polarized laser optical trapping (see [11] and reference therein). Such an experiment should be more easily feasible working with ${ }^{40} \mathrm{~K}$ than with ${ }^{6} \mathrm{Li}$, because in the latter case the electronic spin polarization of the two hyperfine states is very similar at unitarity, due to the large value of the magnetic field required to reach the Feshbach resonance.

Let us finally remark that the formalism developed in this work can be easily generalized to Fermi mixtures of different atomic species (e.g., ${ }^{6} \mathrm{Li}$ and ${ }^{40} \mathrm{~K}$ ). In this case the achievement of an independent tuning of the the two trapping potentials would be much easier.

The authors like to thank F. Chevy and L. P. Pitaevskii for very helpful discussions.

*Electronic address: recati@science.unitn.it

[1] G. B. Partridge, W. Li, R. I. Kamar, Y. Liao, and R. G. Hulet, Science 311, 503 (2006).

[2] M. W. Zwierlein, A. Schirotzek, C. H. Schunck, and W. Ketterle, Science 311, 492 (2006).

[3] M.W. Zwierlein, C.H. Schunck, A. Schirotzek, and W. Ketterle, cond-mat/0605258; Y. Shin, M. W. Zwierlein, C. H. Schunck, A. Schirotzek, and W. Ketterle, condmat/0606432.

[4] J. Carlson and S. Reddy, Phys. Rev. Lett. 95, 060401 (2005); P. Pieri and G. C. Strinati, Phys. Rev. Lett. 96, 150404 (2006); D. E. Sheehy and L. Radzihovsky, ibid. 96, 060401 (2006); J. Kinnunen, L. M. Jensen, and P. Törmä, ibid. 96, 110403 (2006); cond-mat/0604424; M. Haque and H. T. C. Stoof, cond-mat/0601321; W. Yi and L.-M. Duan, Phys. Rev. A 73, 031604(R) (2006); condmat/0604558; K. Machida, T. Mizushime, and M. Ichioka, cond-mat/0604339; C.-H. Pao and S.-K. Yip, cond-mat/ 0604530; M. M. Parish, F. M. Marchetti, A. Lamacraft, and B. D. Simons, cond-mat/0605744.

[5] F. Chevy, Phys. Rev. Lett. 96, 130401 (2006).

[6] T. N. De Silva and E.J. Mueller, Phys. Rev. A 73, 051602(R) (2006); Phys. Rev. Lett. 97, 070402 (2006).

[7] This scenario differs from nuclear physics where the finite value of the symmetry energy is at the origin of the dipole giant resonance [see, e.g., A. Bohr and B. R. Mottelson, Nuclear Structure (W. A. Benjamin, Inc., Reading, MA, 1975), Vol. II].

[8] J. Carlson, S.-Y. Chang, V. R. Pandharipande, and K.E. Schmidt, Phys. Rev. Lett. 91, 050401 (2003); G.E. Astrakharchik, J. Boronat, J. Casulleras, and S. Giorgini, Phys. Rev. Lett. 93, 200404 (2004).

[9] The normal-mixed phase can be included using a more sophisticated many-body approach based on the fixed node quantum Monte Carlo calculation (C. Lobo, A. Recati, S. Giorgini, and S. Stringari, cond-mat/0607730). Similarly to the BCS calculation (Fig. 2) the effects of this additional phase on the dipole polarization are negligible.

[10] In the deep BEC, even for very large displacements of the potentials, the probability for a molecule to dissociate will be small. In fact, the time scale for an adiabatic displacement process has to be larger than the inverse of the minimum gap between the ground and the first excited state. For a molecule in the deep BEC, a simple WKB estimate gives a minimum gap exponentially small for $d \gg a_{\mathrm{ho}}: \delta \simeq \exp \left[-2\left(d / a_{\mathrm{ho}}\right)^{2}\right]$. This result is confirmed by the exact solution of the 2-body problem [Zbignew Idziaszek (private communication)].

[11] I. Carusotto, J. Phys. B 39, S211 (2006). 